\title{
Spontaneous Breast Hematoma Associated With Warfarin
}

\author{
Yasemin Kaya ${ }^{1}$, Ali Bekir Kurt ${ }^{2}$, Ebru Çanakçi ${ }^{3}$, Zeki Yüksel Günaydın ${ }^{4}$, Osman Bektaş ${ }^{5}$, Hasan Öztürk ${ }^{2}$
}

Received: 18.February 2015 Accepted: 4 April 2015/ published online: 17 April 2015

(C) Ordu University Institute of Health Science, Turkey, 2015

\begin{abstract}
Today warfarin sodium (Coumadin) is still the most commonly used agent for anticoagulant therapy. Dosage adjustment is very difficult because of many drugs and food interaction thus it can be very easy to overdose. Coumadin can cause bleeding which can be serious and sometimes lead to death. There are many cases in the literature that caused severe bleeding in different parts of the body but there is not spontaneous breast hematoma. We want to present 84-year-old female patient who had warfarin therapy due to pulmonary embolism, atrial fibrillation and right atrial thrombus. She admitted to the emergency department with a 2 day history of swelling and bruising at left breast without a history of trauma.
\end{abstract}

Key Words: Breast Hematoma, Warfarin, Anticoagulant

\footnotetext{
${ }^{1}$ Ordu University Medical School, Department of Internal Medicine, Ordu, Turkey

${ }^{2}$ Ordu University Medical School, Department of Radiology, Ordu, Turkey

${ }^{3}$ Ordu University Medical School, Department of Anesthesia and Reanimation, Ordu, Turkey

${ }^{4}$ Ordu University Medical School, Department of Cardiology, Ordu, Turkey

${ }^{5}$ Ministry of Health- Ordu Universty Education and Research Hospital, Department of Cardiology, Ordu, Turkey
}

\section{To the editor}

84-year-old female patient admitted to the emergency department with a 2 day history of swelling and bruising at left breast. She was discharged with warfarin therapy due to pulmonary embolism, atrial fibrillation and right atrial thrombus 2 months ago. There was no history of trauma and generalized swelling, edema and common ecchymosis of the left breast (Figure 1) were found on physical examination.

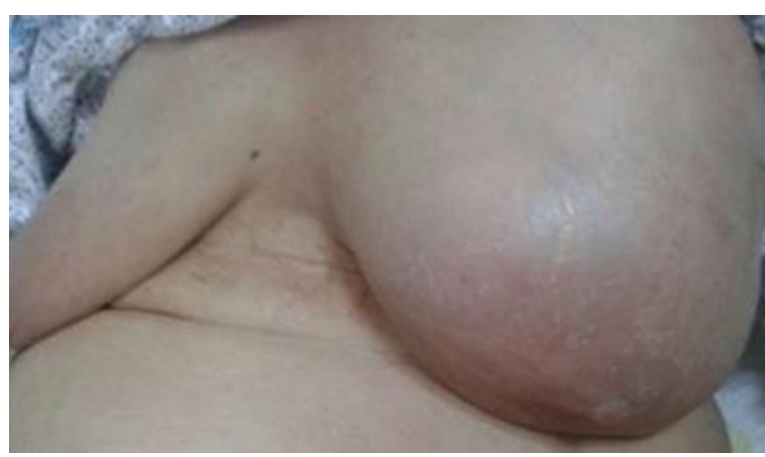

Figure 1: Generalized swelling, edema and common ecchymosis of the left breast 
Other physical examination findings were unremarkable. At the laboratory examination, hemoglobin $(\mathrm{Hb})$ was determined $8.15 \mathrm{~g} / \mathrm{dl}$, prothrombin time (PT) was 58.7 second, internalized normalized ratio (INR) level was 3.3, activated partial thromboplastin time (aPTT) was 50.1 second.

The other laboratory findings were normal. There was edema under the skin and $70 \times 163 \times 124$ millimeter $(\sim 145 \mathrm{ml})$ hematoma which containing hypo-anechoic spaces in the left breast at breast ultrasound (U.S.) (Figure 2).

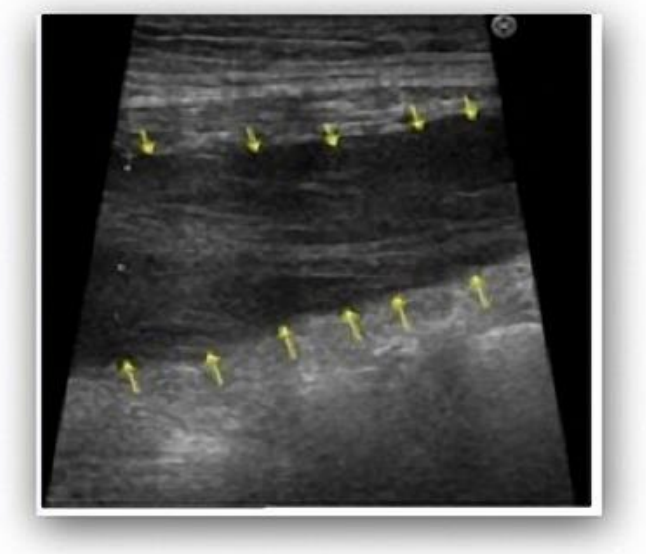

Figure 2: Hematoma in the left breast at breast ultrasound

The patient hospitalized and 4 fresh frozen plasma infusions were administered. In the control laboratory examination $\mathrm{Hb}$ was $5.89 \mathrm{~g} / \mathrm{dl}$, INR was 4.41, PT was 83.3 second, aPTT was 55.3 second. Ecchymosis and swelling were observed to increase at physical examination. It was found that significant increase in the size of the collection left breast ( $\sim 60 \times 190 \times 200 \mathrm{~mm}, \sim 1208 \mathrm{ml})$ at control US. A thoracic computed tomography scan was performed to evaluate expanding hematoma. Fluidfluid showing floor leveling and 145x87x219 millimeter hematoma in the left breast (Figure 3), thickening of the left lateral chest wall and the left breast subcutaneous fatty tissue and increased density of left breast were detected. Collection of muscle was not detected muscle was not detected Patients enrolled in intensive care because of $\mathrm{Hb}$ decline, a significant increase in the volume of the left breast, increase in breast ecchymosis and supportive therapy was continued.

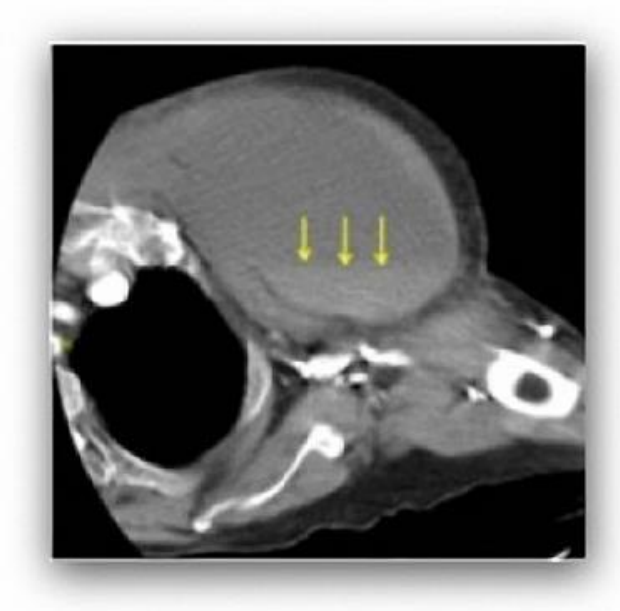

Figure 3: Hematoma in the left breast at thoracic computed tomography

Treatment on the $3 \mathrm{rd}$ day, $\mathrm{Hb}$ was $8 \mathrm{~g} / \mathrm{dl}$, INR was 2, PT was 32 second, aPTT was 51.2 second. Total 24 fresh frozen plasma and 9 unit erythrocyte suspension infusion were administered. Vitamin K and tranexamic acid were applied. Treatment on the $7 \mathrm{rd}$ day blood count began to be stable (Hb: 10 mg/dl, INR: 1.17 PT: $16.8 \mathrm{~min}$ and APTT: 27.9 min). Ultrasonography-guided drainage was performed in patients at 7rd day. Hematoma was $158 \times 73 \times 21 \mathrm{~mm}$ at control US 12 days after drainage. Hematoma had shrunk and from bleeding had stopped drainage catheter. Drainage catheter was pulled. With cardiology department proposal low molecular weight heparin was begun. The patient was consulted to the hematology department and diagnosed as acquired bleeding diathesis and was followed.

Coumadin can cause bleeding which can be serious and sometimes lead to death. Today warfarin sodium is still the most commonly used agent for anticoagulant therapy.

Different results were found in studies with warfarin incidence of bleeding in patients. In one of these studies, It has been reported that incidence of mild or severe bleeding rate between $7.6 \%$ and $16.5 \%$, incidence of severe or life-threatening bleeding rate of 1.3-2.7 / 100 patients / year (Makris et al.,2010). Warfarin bleeding is more common in the elderly. The reason for this comorbid diseases, multiple drug use, and endothelial vascular fragility increase with aging. Our patient was 84 years old. 
Studies in the literature have shown conflicting results regarding the incidence of bleeding in patients on warfarin. In one of these studies while mild or severe bleeding rates were reported between $\% 7,6$ and $\% 16,5$, severe or life threatening bleeding rates were $1,3-2,7 / 100$ patient/year (Makris et al., 2010). Bleeding risk due to warfarin usage is more common in the elderly. The reason for this might be co-morbidity which increased with age, increased vascular and endothelial fragility in elderly and multiple drug use (Beyth and Landefeld, 1995). Besides, the present patient was 84 year old.

The literatures reveal numerous reports of acute spinal hematoma, retroperitoneal hematoma, and rectus sheath hematoma, abdominal wall hematoma, sublingual hematoma, supraglottic hematoma ect. secondary to warfarin use (Fujikawa et al.,2011; Martin et al., 2011; Berthelsen et al.,2013; Yuanyuan, 2014;). However, there was no report about spontaneous breast hematoma

Informed Consent: Written informed consent was obtained from the patient who participated in this case.

Peer-review: Externally peer-reviewed.

Author Contributions: Concept - YK; DesignEÇ; Supervision- YK; Data Collection and/or Processing- ABK; Analysis and/or Interpretation ZYG; Literature Review -OB; Writing -YK; Critical Review -YK

Conflict of Interest: No conflict of interest was declared by the authors.

Financial Disclosure: The authors declared that this study hasn't received no financial support.

\section{References}

Berthelsen RE, Tadbiri S, Rosenstock CV. Spontaneous sublingual haematoma in a patient treated with warfarin. Acta Anaesthesiol Scand. 2013; 57(4):530-1.

Beyth RJ, Landefeld CS. Anticoagulants in older patients. A safety perspective. Drugs Aging 1995; 6(1):45-54.

Fujikawa T, Kawato M, Tanak A. Spontaneous rectus sheath haematoma caused by warfarininduced overanticoagulation. BMJ Case Reports 2011; doi:10.1136/bcr.07.2011.4533

Makris M, Van Veen JJ, Maclean R. Warfarin anticoagulation reversal: management of the asymptomatic and bleeding patient. J Thromb Thrombolysis 2010; 29(2): 171-81.

Martin F, D'Costa H, George G. Supraglottic haematoma in a patient taking warfarin. Emerg Med J. 2011 May; 28(5): 453-4.

Yuanyuan J. Spontaneous abdominal wall hematoma caused by abdominal exercise as a complication of warfarin therapy. Chin Med J 2014; 127 (9): 1796 